\title{
ASSESSMENT OF MANDIBULAR ARCH WIDTH CHANGES IN NON-EXTRACTION CASES AFTER ONE YEAR OF FIXED LINGUAL RETENTION
}

\author{
Dina Hussein El-Ghoul*
}

\begin{abstract}
Introduction: This study was designed to evaluate the changes in mandibular inter-canine inter-premolar and inter-molar width with the presence of a fixed retainer in the mandibular arch for one year in retention using a flexible spiral retainer bonded to all six anteriors.

Methods: Twenty subjects who finished non extraction fixed treatment protocol and completed a one year of a fixed retention regime using a flexible spiral retainer bonded to all mandibular six anteriors. Orthodontic study casts were taken at [T0] pre-treatment, [T1] post-treatment and [T2] one year post-treatment. Orthodontic study cast assessment was done using an electronic caliper.
\end{abstract}

Results: The inter-canine width showed statistically non-significant changes as well as the inter-premolar width changes while the inter-molar width changes was statistically significant.

Conclusions: 1-Fixed retainers proved to stabilize the inter-canine width. 2-The inter-premolar width showed relapse within one year in retention to the pre-treatment measurements. 3-The increase in inter-molar areas was stable to a momentous degree and could be used for increasing the mandibular arch width.

\section{INTRODUCTION}

The recent era of orthodontic treatment experience a shift toward non extraction treatment with the majority of patients refusing to extract sound teeth. In addition to the advent of miniimplants that facilitate distalization, ${ }^{(1)}$ and the introduction of Damon system that claims to alleviate crowding through posterior expansion or adaptation. Allowing the orofacial muscles to align the teeth and determine the arch development; Damon claims that the operator is able to treat non extraction cases without the flaring of anterior teeth or forcing these anterior teeth through the cortical bone as previously associated with aggressive nonextraction techniques. This phenomenon is very similar to the observed "Frankel effect" generated by the Frankel appliance. In his treatment strategy he recommends using a bonded fixed retainer

\footnotetext{
* Lecturer, Orthodontic Department. Faculty of Oral and Dental Medicine, Ain Shams University, Cairo, Egypt.
} 
[0.016 x 0.022 Bond-a-Braid (Reliance) wire] bonded to the palatal surface of the four maxillary anterior teeth and [0.026 round stainless steel wire] adapted to the lingual surface of the mandibular six anteriors. ${ }^{(2)}$ In the same time, a lot of adults are seeking orthodontic treatment with the aesthetics being their main concern that lays fixed retention as the rule rather than an exception. And this brought up the exclamation of whether a fixed retainer would be enough to guarantee stability of treatment changes in posterior arch width or not? The aim of this study was to evaluate the changes in intercanine, inter-premolar and inter-molar width with the presence of a fixed retainer in the mandibular arch for one year in retention using a flexible spiral retainer bonded to all six anteriors.

\section{MATERIALS AND METHODS}

The sample consisted of twenty subjects who finished non extraction fixed treatment protocol and completed a one year of a fixed retention regime using a flexible spiral retainer bonded to all mandibular six anteriors fig1. All subjects were selected from the Outpatient Clinic of the Orthodontic Department Faculty of Dentistry, Ain

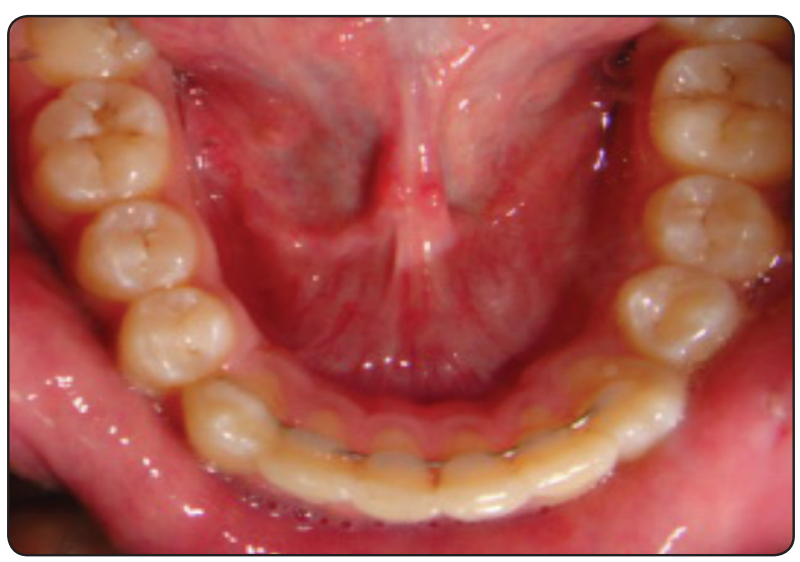

Fig (1) Flexible spiral retainer bonded to all mandibular six anteriors
Shams University. All subjects aged from 20-25 years, had class I molar and canine relationship, free from abnormal habits (attrition, abrasion, erosion or bruxism). Did not perform any interproximal enamel reduction or circumferential supracrestal fiberotomy. All subjects were free from any dental abnormalities and all had good oral hygiene. Orthodontic study casts were taken at (T0) pretreatment, (T1) post-treatment and (T2) one year in retention. Orthodontic study cast assessment was done using an electronic caliper with very sharp and fine peak edge to allow easiness in access and assure accurate measurements of $0.01 \mathrm{~mm}$.

The following measurements were made to the nearest $0.01 \mathrm{~mm}$ at $\mathrm{T} 0, \mathrm{~T} 1$ and $\mathrm{T} 2$. Fig 2

1) Inter-canine width: The distance between canine cusp tips or estimated cusp tips in cases of wear facets.

2) Inter-premolar width: measured as the distance between the facial contact point between the first and second premolars

3) Inter-molar width: measured as the distance between the disto-buccal cusp tips of the first permanent molars. ${ }^{(3)}$

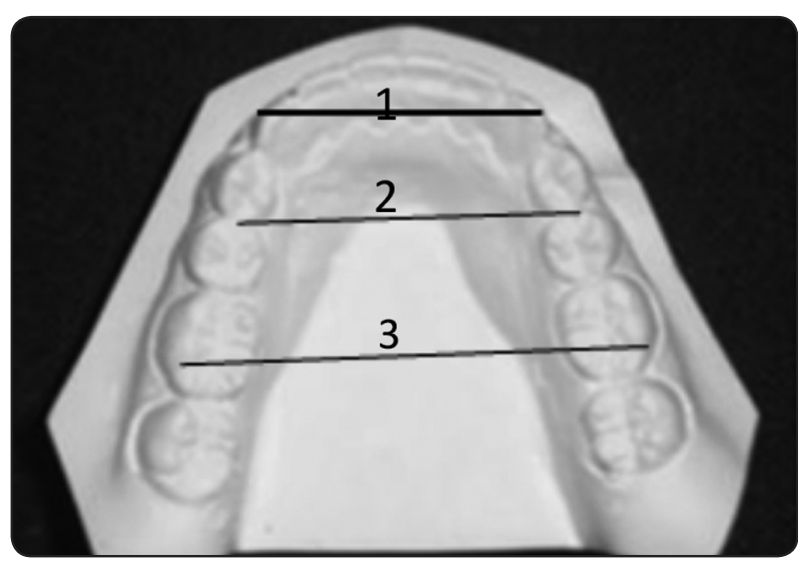

Fig (2) Mandibular orthodontic study cast for a subject with a flexible spiral retainer for one year. Showing: 1: Intercaninewidth 2:Inter-premolar width 3: Inter-molar width. 


\section{Statistical analysis}

All measurements were presented into an excel spreadsheet and analyzed using a statistical software package (SPSS version 17.0, Chicago, III) for windows. Descriptive statistics minimum, maximum, mean and standard deviation of the inter-canine, inter-premolar and inter-molar width at T0, T1 and T2 was done. Normal distribution of the data was tested using Kolmogorov-Smirnov test and it revealed that the data is normally distributed. Hence parametric tests will be applied. One Way Repeated Measure ANOVA was used to compare the mean inter-canine, inter-premolar and inter-molar width changes at $\mathrm{T} 0, \mathrm{~T} 1$ and $\mathrm{T} 2$, followed by Bonferroni Method for multiple comparisons to compare between different time intervals within each measurement. Significant level was set at $\mathrm{P}<0.05$ and when $\mathrm{P}<0.01$ it's considered as highly significant.

\section{RESULTS}

\section{Inter-canine width}

Descriptive statistics minimum, maximum, mean and standard deviation of the inter-canine width is shown in table (1).

Table (2) and figure 3 showed a statistically non significant difference between the inter-canine width in T0, T1 and T2.

TABLE (1): Descriptive statistics minimum, maximum, mean and standard deviation of the inter-canine width at T0,T1 and T2.

\begin{tabular}{|c|c|c|c|c|}
\hline & Minimum & Maximum & Mean & Std. Deviation \\
\hline T0 & 25.00 & 29.30 & 27.00 & 1.40 \\
\hline T1 & 25.00 & 28.60 & 26.87 & 1.40 \\
\hline T2 & 25.00 & 28.70 & 26.80 & 1.31 \\
\hline
\end{tabular}

TABLE (2) One Way Repeated Measure ANOVA to compare the mean inter-canine width changes at $\mathrm{T} 0, \mathrm{~T} 1$ and $\mathrm{T} 2$.

\begin{tabular}{|c|c|c|c|c|c|}
\hline & & Mean & $\begin{array}{c}\text { Std. } \\
\text { Deviation }\end{array}$ & F & P value \\
\hline \multirow{2}{*}{$\begin{array}{c}\text { Inter-canine } \\
\text { width }\end{array}$} & T0 & 27.00 & 1.40 & & \\
\cline { 2 - 4 } & T1 & 26.87 & 1.40 & \multirow{2}{*}{0.93} & 0.42185 \\
\cline { 2 - 4 } & T2 & 26.80 & 1.31 & & \\
\hline
\end{tabular}

$P>0.05$ Non Significant NS

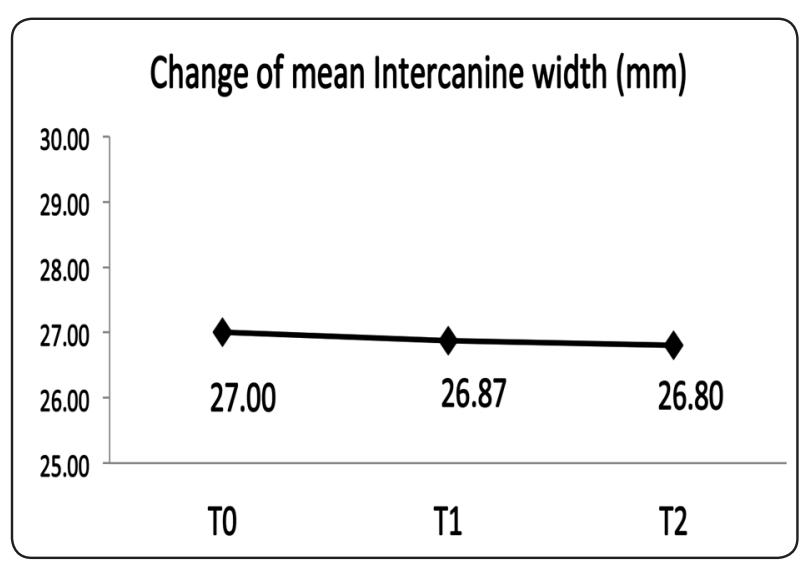

Fig. (3) Changes in inter-canine width at T0, T1 and T2.

\section{Inter-premolar width:}

Descriptive statistics minimum, maximum, mean and standard deviation of the inter-premolar width is shown in table (3).

Table (4) and figure 4 showed a statistically highly significant difference between the interpremolar width in $\mathrm{T} 0, \mathrm{~T} 1$ and $\mathrm{T} 2 . \mathrm{P}<0.01$

Table (5) illustrates a highly significant difference when comparing $\mathrm{T} 2$ and $\mathrm{T} 1 \mathrm{P}<0.01$, while the difference between $\mathrm{T} 1$ and $\mathrm{T} 0$ was less significant $\mathrm{P}<0.05$. At the end the difference between $\mathrm{T} 0$ and $\mathrm{T} 2$ was statistically insignificant.

TABLE (3): Descriptive statistics minimum, maximum, mean and standard deviation of the inter-premolar width at $\mathrm{T} 0, \mathrm{~T} 1$ and $\mathrm{T} 2$.

\begin{tabular}{|c|c|c|c|c|}
\hline & Minimum & Maximum & Mean & Std. Deviation \\
\hline T0 & 33.00 & 37.80 & 36.10 & 1.66 \\
\hline T1 & 36.80 & 39.00 & 37.70 & 0.94 \\
\hline T2 & 35.40 & 38.10 & 36.80 & 1.07 \\
\hline
\end{tabular}


TABLE (4) One Way Repeated Measure ANOVA to compare the mean inter-premolar widths changes at $\mathrm{T} 0, \mathrm{~T} 1$ and $\mathrm{T} 2$.

\begin{tabular}{|c|c|c|c|c|c|}
\hline & & Mean & $\begin{array}{c}\text { Std. } \\
\text { Deviation }\end{array}$ & F & P value \\
\hline \multirow{2}{*}{$\begin{array}{c}\text { Inter- } \\
\text { premolar } \\
\text { width }\end{array}$} & T0 & 36.10 & 1.66 & & \\
\cline { 2 - 4 } & T1 & 37.70 & 0.94 & 10.72 & 0.00213 \\
\cline { 2 - 4 } & T2 & 36.80 & 1.07 & & \\
\hline
\end{tabular}

$P<0.01$ HS

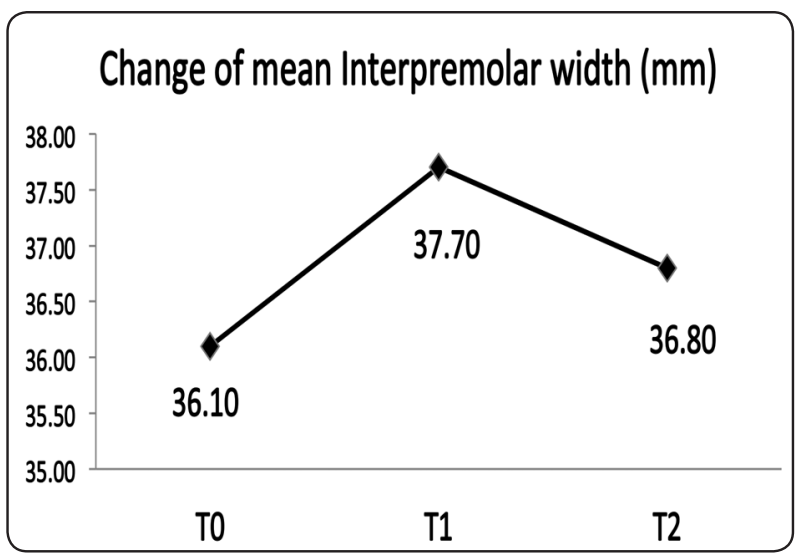

Fig. (4) Changes in inter-premolar width at T0,T1 and T2

TABLE (5): Bonferroni Method for multiple comparisons to detect the inter-premolar width changes within each time interval at $\mathrm{T} 0, \mathrm{~T} 1$ and $\mathrm{T} 2$.

\begin{tabular}{|c|c|c|c|c|}
\hline & & $\begin{array}{c}\text { Mean } \\
\text { Difference }\end{array}$ & $\begin{array}{c}\text { Std. } \\
\text { Error }\end{array}$ & P Value \\
\hline \multirow{2}{*}{$\begin{array}{c}\text { Inter- } \\
\text { premolar } \\
\text { width }\end{array}$} & T1 - T0 & 1.60 & 0.47 & 0.04289 \\
\cline { 2 - 5 } & T2 - T0 & 0.70 & 0.33 & 0.22891 \\
\cline { 2 - 5 } & T2 - T1 & -0.90 & 0.18 & 0.00762 \\
\hline
\end{tabular}

\section{Inter-molar width:}

Descriptive statistics minimum, maximum, mean and standard deviation of the inter-molar width is shown in table (6).
Table (7) and figure (5) showed a statistically highly significant difference between the interpremolar width in T0, T1 and T2. $\mathrm{P}<0.01$

Table (8) illustrates a highly significant difference when comparing $\mathrm{T} 0$ and $\mathrm{T} 1 \mathrm{P}<0.01$, while the difference between $\mathrm{T} 2$ and $\mathrm{T} 1$ was less significant $\mathrm{P}<0.05$. At the end the difference between $\mathrm{T} 0$ and $\mathrm{T} 2$ was considered to be statistically significant.

TABLE (6) Descriptive statistics minimum, maximum, mean and standard deviation of the inter-molar width at T0,T1 and T2.

\begin{tabular}{|l|c|c|c|c|}
\hline & Minimum & Maximum & Mean & Std. Deviation \\
\hline T0 & 42.00 & 48.80 & 46.29 & 2.12 \\
\hline T1 & 42.80 & 50.00 & 47.36 & 2.40 \\
\hline T2 & 42.20 & 49.00 & 46.80 & 2.29 \\
\hline
\end{tabular}

TABLE (7) One Way Repeated Measure ANOVA to compare the mean inter-molar widths changes at $\mathrm{T} 0, \mathrm{~T} 1$ and $\mathrm{T} 2$.

\begin{tabular}{|c|c|c|c|c|c|}
\hline & & Mean & $\begin{array}{c}\text { Std. } \\
\text { Deviation }\end{array}$ & F & P value \\
\hline \multirow{2}{*}{$\begin{array}{c}\text { Inter- } \\
\text { molar } \\
\text { width }\end{array}$} & T0 & 46.29 & 2.12 & & \\
\cline { 2 - 4 } & T1 & 47.36 & 2.40 & \multirow{2}{*}{17.15} & 0.00030 \\
\cline { 2 - 4 } & T2 & 46.80 & 2.29 & & \\
\hline
\end{tabular}

$P<0.01$ H S

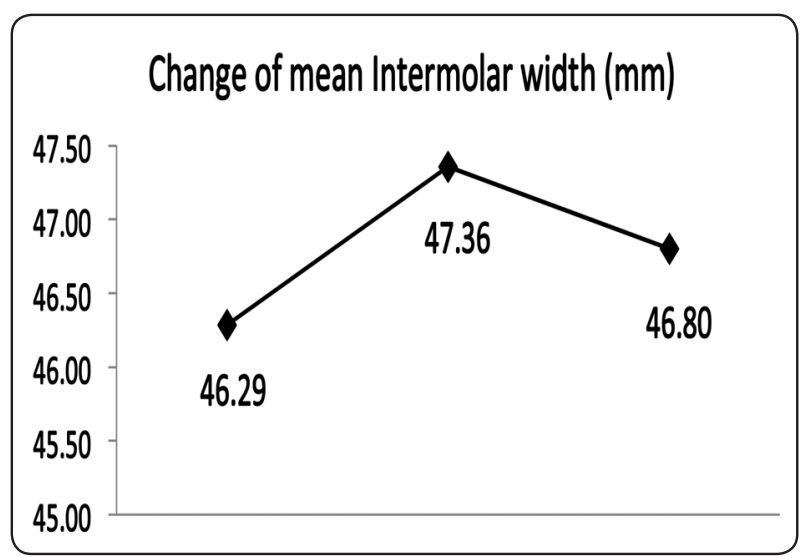

Fig. (5) Changes in inter-molar width at T0,T1 and T2. 
TABLE (8) Bonferroni Method for multiple comparisons to detect the inter-molar width changes within each time interval at $\mathrm{T} 0, \mathrm{~T} 1$ and $\mathrm{T} 2$.

\begin{tabular}{|c|c|c|c|c|}
\hline & & $\begin{array}{c}\text { Mean } \\
\text { Difference }\end{array}$ & $\begin{array}{c}\text { Std. } \\
\text { Error }\end{array}$ & P Value \\
\hline \multirow{2}{*}{$\begin{array}{c}\text { Inter- } \\
\text { molar } \\
\text { width }\end{array}$} & T1 - T0 & 1.07 & 0.21 & 0.00704 \\
\cline { 2 - 5 } & T2 - T0 & 0.51 & 0.17 & 0.06472 \\
\cline { 2 - 5 } & T2 - T1 & -0.56 & 0.17 & 0.04588 \\
\hline
\end{tabular}

$$
P<0.05 S \quad P>0.05 N S \quad P<0.01 H S
$$

\section{DISCUSSION}

Mandibular arch crowding is a very common and highly challenging orthodontic problem, as its extent is a determining factor in either to extract or not. Nowadays non-extraction orthodontic treatment comes to be a very popular choice for the patients and orthodontists as well. Gaining space in the mandibular arch has been a limiting factor for the belief that the expansion of the arch is not stable. Thus the use of permanent retainer became a must and due to the increase in the esthetic awareness and demand fixed lingual retainers are widely used. For that this study was dedicated to assess the changes in the mandibular arch width in non- extraction cases one year post treatment and during retention with fixed lingual retainer.

As teeth erupt into the mouth, they are guided into a specific zone of neutrality that determines the horizontal position of each tooth in the arch. The neutral zone is where the opposing forces of the tongue and cheek are equal. The boundaries of the neutral zone form a matrix for the dental arches. Any attempt to move any part of the dental arch, including the alveolar structures outside the neutral zone, will result in increased pressure against the part that intrudes. The neutral zone has not been given enough importance in the literature, but as a determinant of occlusion it cannot be ignored. Understanding of the neutral zone makes it readily apparent why so many orthodontic results do not remain stable. The strength of contractile force of the Buccinators, Orbicularis Oris bands and its length forms an inviolate outer limit for arch size. ${ }^{(4)}$

Reidel ${ }^{(5)}$ stated that arch form, particularly in the mandibular arch, could not be altered by appliance therapy. Inter-canine and inter-molar widths tend to decrease during the post retention period, especially when expanded during treatment. ${ }^{(5,6)}$

Housley et al (7) conducted a study on the stability of transverse expansion in the mandibular arch and they concluded that only $8 \%$ of the arch width increase at the canines was sustained after retention, but, at the first and second premolars and the first molars, about $60 \%$ to $70 \%$ of the expansion remained. It seems that the stability of expansion in the canine region could not be sustained. On the other hand; the increase in the premolar and molar areas was stable to a significant degree and could be used for gaining arch perimeter.

However the results of this study showed statistically non-significant changes regarding the inter-canine width this cast some doubts on the assumption that non-extraction protocols can increase all the mandibular arch widths. And aroused an important question, whether changes in the mandibular width happen in the posterior mandibular arch dimensions only. Or the changes in the inter-canine width are highly susceptible to relapse and this is in agreement with Reidel ${ }^{(5)}$ and Housley et al ${ }^{(7)}$. This inquiry recommends further studies. As for the inter-premolar width results of the current study revealed statistical significant increase during the treatment period (T0-T1), followed by statistically high significant decrease after treatment and during retention (T1-T2) and this resulted in statistically non-significant changes in the interpremolar width after one year of fixed lingual 
retention (T0-T2) this finding is in accordance with the postulation of Reidel ${ }^{(5)}$ and contradict that of Housley et al ${ }^{(7)}$. The inter-molar width revealed statistically high significant increase during the treatment period (T0-T1), followed by statistically significant decrease after treatment and during retention (T1-T2) and this resulted in statistically significant increase in the inter-molar width after one year of fixed lingual retention (T0-T2) this finding agrees with Housley et al ${ }^{(7)}$ and challenges Reidel (5) declaration. This debate should be considered and furthermore studies should be planned to support or refuse the rising idea of non-extraction orthodontic treatment and the increasing demand of the different types of fixed lingual retainers.

A lot of factors should be considered before deciding our final treatment plan or retention protocol, by putting the end in mind and to avoid an inevitable relapse, the amount of expansion should be evaluated along with the length and strength of the three bands of the buccinator muscle, tongue size and pressure, the size of the mouth must also be evaluated when a change in arch size is being contemplated. A very small orifice is far more restrictive than a large broad opening that exposes the dentition all the way around to the molars.

\section{CONCLUSION}

1) Fixed retainers proved to stabilize the inter-canine width.

2) The inter-premolar width showed relapse within one year in retention to the pre-treatment measurements.

3) The increase in inter-molar areas was stable to a momentous degree and could be used for increasing the mandibular arch width.

\section{REFERENCES}

1. Creekmore TD and Eklund MK. The possibility of skeletal anchorage. J Clin Orthod 1983; 17:266-269.

2. Damon Dwight. Damon System The Work Book. blog. ortodonti.ro/wp-content/uploads/2013/07/Damon-System.

3. Aluko IA, daCosta OO and Isiekwe MC . Dental arch widths in the early and late permanent dentitions of a Nigerian population, Nig Dent J 2009;17:1 Jan - June .

4. Peter E. Dawson.The neutral zone. In: Functional Occlusion: From TMJ to Smile Design, St. Louis: Elsevier Inc.2007; p. 131-140,

5. Reidel RA. A review of the retention problem. Angle Orthod 1960; 30:179-194.

6. Shapiro PA. Mandibular dental arch form and dimension. Am J Orthod 1974; 66:58-70.

7. Housley Jeffrey A., Nanda Ram S., Currier G. Frans and McCune Dale E.Stability of transverse expansion in the mandibular arch .Am J Orthod Dentofacial Orthop 2003;124:288-293. 